EPJ Web of Conferences 47, 06006 (2013)

DOI: $10.1051 /$ epjconf/20134706006

(C) Owned by the authors, published by EDP Sciences, 2013

\title{
A 2 epoch proper motion catalogue from the UKIDSS Large Area Survey
}

\author{
Leigh Smith ${ }^{1}$, a , Phil Lucas ${ }^{1}$, Ben Burningham ${ }^{1}$, Hugh Jones ${ }^{1}$, David Pinfield ${ }^{1}$, \\ Ricky Smart ${ }^{2}$ and Alexandre Andrei ${ }^{3}$
}

\author{
${ }^{1}$ Centre for Astrophysics Research, Science and Technology Research Institute, University of \\ Hertfordshire, Hatfield AL10 9AB, UK \\ 2 INAF/Osservatorio Astronomico di Torino, Strada Osservatorio 20, 10025 Pino Torinese, \\ Italy \\ ${ }^{3}$ Observatório Nacional/MCT, R. Gal. José Cristino 77, CEP20921-400, RJ, Brazil
}

\begin{abstract}
The UKIDSS Large Area Survey (LAS) began in 2005, with the start of the UKIDSS program as a 7 year effort to survey roughly 4000 square degrees at high galactic latitudes in $\mathrm{Y}, \mathrm{J}, \mathrm{H}$ and $\mathrm{K}$ bands. The survey also included a significant quantity of 2-epoch $\mathrm{J}$ band observations, with epoch baselines ranging from 2 to 7 years. We present a proper motion catalogue for the 1500 square degrees of the 2 epoch LAS data, which includes some 800,000 sources with motions detected above the $5 \sigma$ level. We developed a bespoke proper motion pipeline which applies a source-unique second order polynomial transformation to UKIDSS array coordinates of each source to counter potential local non-uniformity in the focal plane. Our catalogue agrees well with the proper motion data supplied in the current WFCAM Science Archive (WSA) DR9 catalogue where there is overlap, and in various optical catalogues, but it benefits from some improvements. One improvement is that we provide absolute proper motions, using LAS galaxies for the relative to absolute correction. Also, by using unique, local, 2nd order polynomial tranformations, as opposed to the linear transformations in the WSA, we correct better for any local distortions in the focal plane, not including the radial distortion that is removed by their pipeline.
\end{abstract}

\section{INTRODUCTION}

Stellar proper motion is the angular movement of a star in a given time period. All stars have some component of motion due to their motion around the Galaxy and "gravitational kicks" they may have received through interaction with other massive objects. Motion perpendicular to a line between the star and the observer is the proper motion, which can be measured through careful observation of its position over two or more epochs, given sufficient time between observations.

Current surveys are mostly optical in nature (or contain at least an optical component) owing to their need for a large epoch baseline usually meaning optical photographic plates are used for at least the first epoch. Our catalogue is one of very few wide field IR proper motion surveys and can be expected to reveal objects not detected in optical surveys while also providing kinematic data for known objects than can serve many scientific purposes, such as investigating the ages of T dwarfs (Smith et al., in prep.).

\footnotetext{
ae-mail: 1.smith10@herts.ac.uk
}

This is an Open Access article distributed under the terms of the Creative Commons Attribution License 2.0, which permits unrestricted use, distribution, and reproduction in any medium, provided the original work is properly cited. 


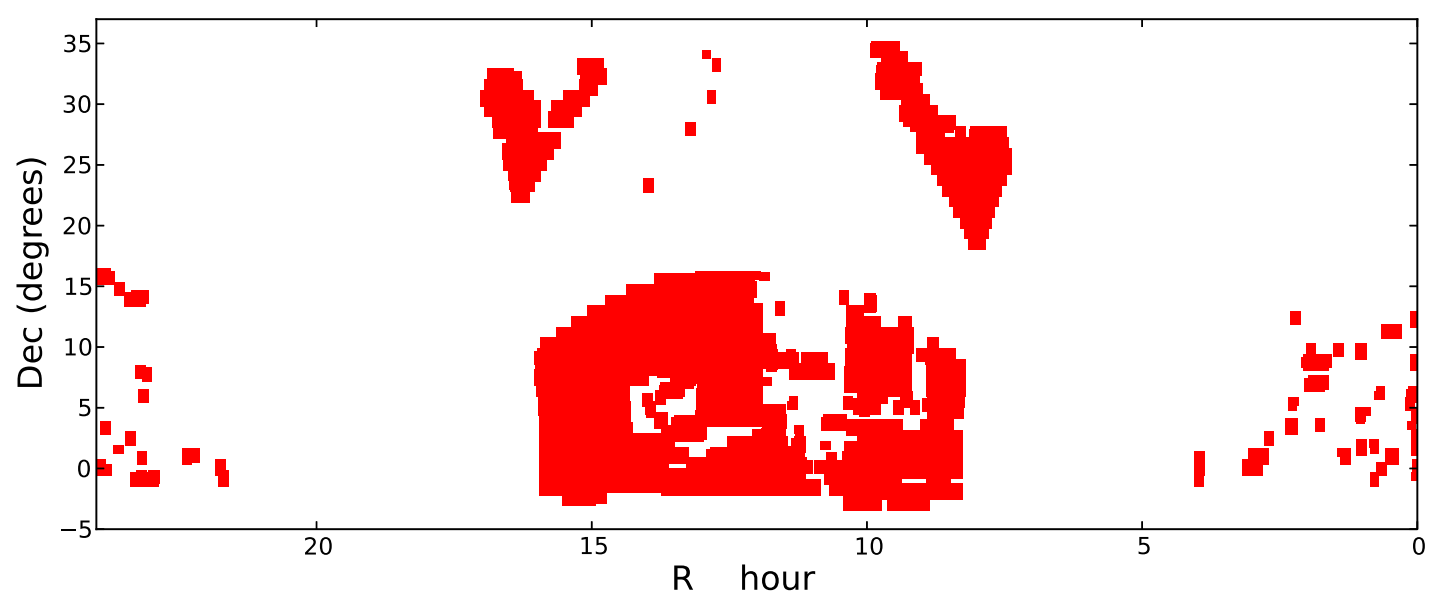

Figure 1. Our UKIDSS LAS proper motion catalogue sky coverage.

\section{METHOD}

The LAS covers 4028 square degrees in YJHK passbands to an approximate depth of 19.6 in $\mathrm{J}$ and is complemented in ugriz optical passbands by the Sloan Digital Sky Survey (SDSS). It included a second epoch of observations in the J passband to calculate proper motions and investigate stellar variability. In the final months of the UKIDSS program great effort was made to observe as much as possible at second epoch. The final second epoch coverage and the footprint of our catalogue is around 1500 square degrees (see Fig. 1). We use a match limit of 6" and with a minimum epoch baseline of $\sim 2$ years, giving us a hard limit on proper motion detections of $3 \operatorname{arcsec}_{\mathrm{yr}}{ }^{-1}$.

Our data set contains $\sim 17$ Million sources separated into $\sim 33,00013 \times 13$ arcminute frames. We process each frame individually, producing global (whole-frame) and local second order polynomial transforms by fitting relatively static star array positions between epochs. A local polynomial is fit using reference stars from within a smaller radius than the frame. The second epoch array positions are corrected to the first epoch frame using the calculated transform. We then fit first epoch source array positions to their first epoch equatorial tangent plane positions using a third order polynomial. Subtracting the first from corrected second epoch positions calculates source motions, dividing these by the epoch baseline gives us their proper motions. We use locally transformed positions to calculate proper motions where possible due to their lower uncertainties on average. We correct our relative proper motions to absolute by subtracting the median proper motion of sources flagged as galaxies within 3 degrees with small proper motion uncertainties.

\section{RESULTS}

We compared our absolute proper motions to those two existing large baseline optical proper motion catalogues, the revised NLTT catalogue of Salim and Gould (2003) [1] and the LSPM-n catalogue of Lépine and Shara (2005) [2]. We matched the catalogues using 3" separation and $0.5 \mathrm{~J}$ magnitude variation tolerances and find 2,381 and 419 matches in the LSPM and rNLTT catalogues respectively (see Fig. 2). The data are very well correlated with Pearson's r values of 0.99 and 0.98 for rNLTT and LSPM respectively. This is encouraging since both catalogues benefit from typical epoch baselines an order of magnitude greater than ours. Typical absolute proper motion uncertainties for our results are 10

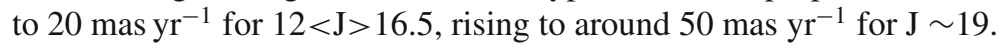



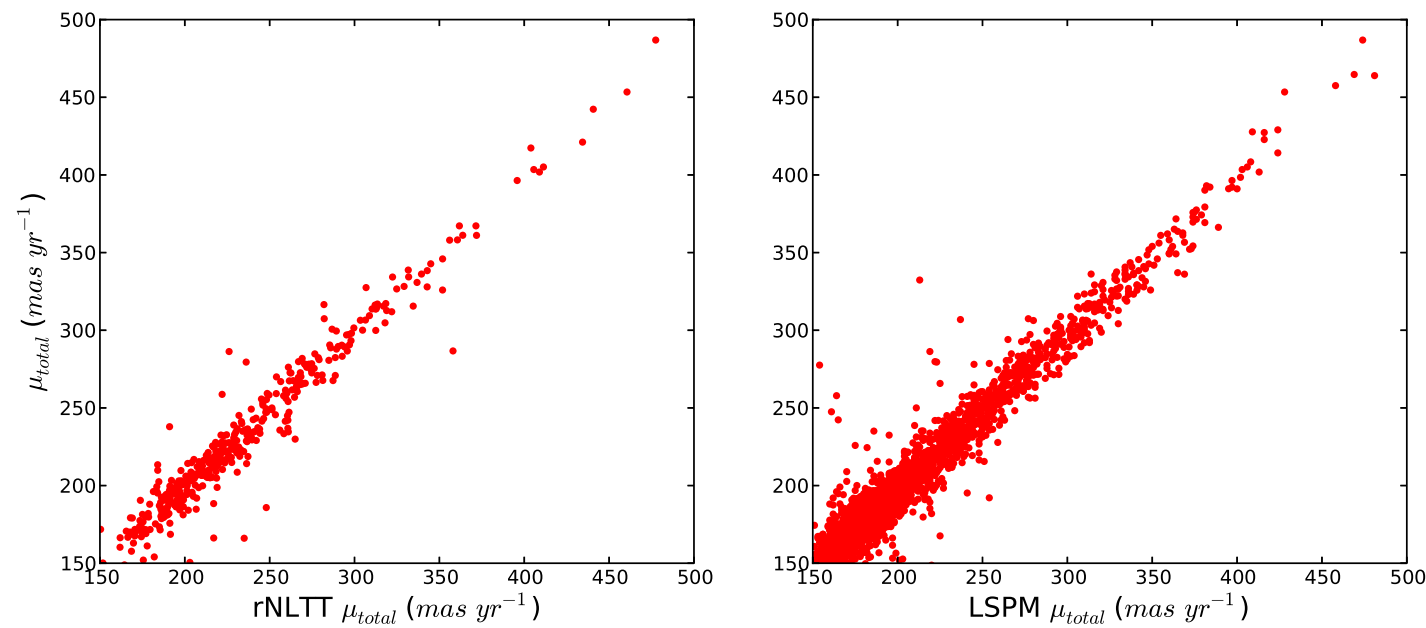

Figure 2. Comparison between our proper motion values (vertical axis) and rNLTT and LSPM proper motion values. We find the data are well correlated (Pearson's r 0.99 and 0.98 for rNLTT and LSPM respectively) and agree within their uncertainties to the degree expected.

\subsection{Objects of interest}

A 260 square degree subsection (the WSA LAS DR9 overlap area) has been briefly scrutinized for interesting objects, including cool white dwarfs and ultracool dwarfs (benchmark objects in particular). We refer the reader to Catalan, et al. (2012) [3] for detailed analysis of the brightest Pure H ultra cool white dwarf (effective temperature $3880 \pm 90 \mathrm{~K}$ ) which was apparent in our catalogue due to it's high proper motion. We also refer the reader to Burningham, et al. (2013, in prep), a search for UKIDSS LAS benchmark $\mathrm{T}$ dwarfs using our catalogue among others.

\section{SUMMARY}

We have produced a 1500 square degree proper motion catalogue using two epochs of NIR UKIDSS

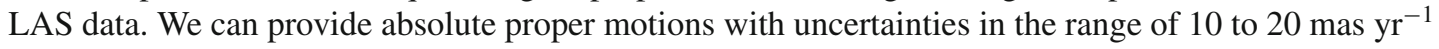
for $12<J>16.5$. The catalogue will be made publicly available during 2013 , until this time we encourage the reader to contact and collaborate with the authors if our catalogue may be of use to them.

\section{References}

[1] Salim, S. and Gould, A., The Astrophysical Journal 582, 1011-1031 (2003)

[2] Lépine, S. and Shara, M. M., The Astronomical Journal 129, 1483-1522 (2005)

[3] Catalan, et al., Astronomy \& Astrophysics 546 (2012) 